\title{
甲醇低温脱氢均相过渡金属催化剂研究进展
}

\author{
张翔郭彩红* 武海顺 \\ (山西师范大学化学与材料科学学院 磁性分子与磁信息材料教育部重点实验室 临汾 041004)
}

\begin{abstract}
摘要 能源紧缺和环境污染已成为世界所面临的重大难题, 开发绿色能源、寻找化石燃料的替代品迫在眉睫. 甲醇由 于其含氢密度高、结构简单、环境友好等优点成为未来储氢的理想载体. 降低甲醇脱氢反应温度, 提高反应选择性, 研 发均相甲醇脱氢催化剂一直受到国内外的广泛关注. 本文围绕甲醇热脱氢和甲醇水相重整, 总结了近年来报道的过渡 金属均相催化剂反应体系, 重点介绍了钉、铑、铱、铁、锰基络合物的结构特点、催化甲醇脱氢条件、反应产率以及 催化机理, 分析比较了催化剂在使用过程中的活性差异, 并对进一步提高水相重整催化剂性能做了展望.
\end{abstract}

关键词 甲醇; $\mathrm{C}-\mathrm{H}$ 键活化; 脱氢反应; 均相催化剂; 钉

\section{Recent Developments of Homogeneous Transition-Metal Catalysts for Low Temperature Dehydrogenation of Methanol}

\author{
Zhang, Xiang Guo, Caihong* Wu, Haishun \\ (Key Laboratory of Magnetic Molecules, Magnetic Information Materials, Ministry of Education, \\ School of Chemical and Material Science, Shanxi Normal University, Linfen 041004)
}

\begin{abstract}
It is extremely urgent to develop green energy and find alternatives to fossil fuels since the energy shortage and environmental pollution become the worldwide concern. Methanol has emerged as a promising carrier for hydrogen storage owing to its high hydrogen density, simple structure and environmental friendly substance. The methanol reforming has triggered great efforts on the development of homogeneous catalysts, and the aim is to decrease methanol dehydrogenation reaction temperature and improve the selectivity. This review summarizes the recent research progresses in the homogeneously transition-metal catalyzed thermal dehydrogenation of methanol and aqueous methanol reforming. It mainly focuses on the structural characteristics of $\mathrm{Ru}, \mathrm{Rh}, \mathrm{Ir}, \mathrm{Fe}$ and Mn-based complexes, catalytic reaction conditions, the reaction yield, and the catalytic reaction mechanism. The differences in the reactivities of these catalysts are analyzed and compared. Not only a summary is given, but also some perspectives and inspiration for improving the performance of aqueous methanol reforming catalysts for future research are discussed.

Keywords methanol; $\mathrm{C}-\mathrm{H}$ activation; dehydrogenation; homogeneous catalyst; $\mathrm{Ru}$
\end{abstract}

全球能源 $80 \%$ 以上是化石燃料 ${ }^{[1]}$, 如果继续维持这 样的能源供给, 石油资源将会在本世纪末消耗殆尽. 氢 气是最理想的清洁能源, 燃烧会释放大量的热, 其唯一 产物是无污染的水 ${ }^{[2]}$. 风能、太阳能和水电能技术虽然 已经发展了 50 多年, 但由于时间和地理位置的局限性, 不能持续性供能 ${ }^{[3]}$, 而 “氢经济” 概念 ${ }^{[4]}$ 的提出弥补了这 些不足, 并提供了一个有希望的解决方案. 然而, 氢气 在室温下是易燃易爆气体, 极易溢出容器, 体积能量密 度极低, 这使得其在运输和存储时必须经过高压压缩 $(35 \sim 70 \mathrm{MPa})$ 或低温 $\left(-253{ }^{\circ} \mathrm{C}\right)$ 液化, 所以氢气本身并
不是一种理想的能源载体, 这是发展 “氢经济” 的一大 难题.

现如今储氢主要有高压气态储氢技术 ${ }^{[5]}$ 、低温液化 储氢技术 ${ }^{[6]}$ 、吸附储氢技术 ${ }^{[7]}$ 、水合物储氢技术 ${ }^{[8]}$ 和化学 储氢技术 ${ }^{[9]}$ 等 $^{[10]}$. 前两种物理储氢技术成本低、易放氢, 但安全性较差. 吸附储氢技术受吸附剂影响较大 ${ }^{[11]}$, 其 优点是速度快、完全可逆和循环性好 ${ }^{[12]}$, 但通常需要在 低温和高压下才能达到合适的存储容量 ${ }^{[13]}$. 水合物储 氢技术易脱氢、成本低、能耗低, 但其储氢密度较低 ${ }^{[10]}$. 化学储氢方法是固态或液态化合物通过适当的脱氢反

\footnotetext{
* Corresponding author. E-mail: sxgch2006@163.com

Received February 26, 2019; revised March 27, 2019; published online April 11, 2019.

Project supported by the National Natural Science Foundation of China (No. 21373131).

国家自然科学基金(No. 21373131)资助项目.
} 
应(最好是完全可逆的)生成稳定的化合物来释放氢气. 作为氢载体必须要考虑以下几个标准: 含氢量 $\left(\mathrm{H}_{2}\right.$ 的质 量分数 $w)$ 、氢体积容量 $\left(\mathrm{H}_{2} /\right.$ 载体: 单位为 $\left.\mathrm{kg} / \mathrm{m}^{3}\right)$ 和脱氢 效率 ${ }^{[14]}$. 现已报道的固体载体有纯金属氧化物、金属氧 化物合金 ${ }^{[15]}$ 、金属硼氢化物 ${ }^{[16]}$ 、氨硼烷 ${ }^{[17]}$ 等. 液态有机 氢载体 $(\mathrm{LOHCs})$ 主要包括环己烷衍生物和杂环化合 物 ${ }^{[18]}$ 、氨 ${ }^{[19]} 、$ 肼 ${ }^{[20]}$ 和氨硼烷 ${ }^{[17]} 、 2$-甲基噻吩 ${ }^{[21]}$ 、甲酸 ${ }^{[22]}$ 和醇类 ${ }^{[23]}$. 其中甲醇的含氢量为 $12.6 \%$, 室温下 $1 \mathrm{~m}^{3}$ 甲 醇可容纳 $99.77 \mathrm{~kg}$ 氢气, 脱氢效率约 $90 \%$ 以上. 此外甲 醇价格便宜, 在室温下为稳定的液体, 环境条件下挥发 性低、溶解性好, 具有使用现有基础设施存储和运输的 可能性，备受人们的关注，是一种很有应用潜力的储氢 燃料. 在二十世纪末期, Asinger ${ }^{[24]}$ 提出了甲醇经济的概 念(图 1), 即二氧化碳加氢生成甲醇以及甲醇脱氢为二 氧化碳的碳平衡系统. 根据使用底物分子中不同类型的 氢原子，甲醇脱氢反应可以分为三类: 第一类为仅利用 甲醇的两个氢原子, 脱氢生成甲醛和氢气(表 1, 反应式 1); 第二类为甲醇的四个氢原子全部放出, $1 \mathrm{~mol}$ 甲醇脱 氢为 $1 \mathrm{~mol}$ 一氧化碳和 $2 \mathrm{~mol}$ 氢气(表 1, 反应式 2 和 $3^{[25]}$; 第三类为水中的氢原子共同参与反应, 这使得 1 $\mathrm{mol}$ 甲醇脱氢为 $1 \mathrm{~mol} \mathrm{CO}_{2}$ 和 $3 \mathrm{~mol} \mathrm{H}_{2}$ (表 1, 反应式 4 和 5), 其中 $1 \mathrm{~mol} \mathrm{H}_{2}$ 来自水. 以上所有反应都是吸热反应. 通过比较表 1 中甲醇的五种脱氢反应, 可以看出反应式 1 的氢气利用率低, 反应式 2 和 3 会产生有毒性的 $\mathrm{CO}$, 所以反应 $1 \sim 3$ 都是不可取的. 反应式 4 和 5 是人们研究 甲醇经济的焦点.

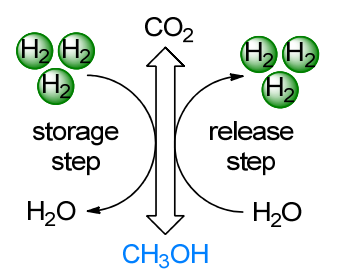

图 1 基于甲醇/ $\mathrm{CO}_{2}$ 的可逆储氢循环

Figure 1 Reversible hydrogen storage cycle based on the methanol $/ \mathrm{CO}_{2}$ couple

表 1 基于甲醇/ $\mathrm{CO}_{2}$ 可逆储氢的热力学数据 ${ }^{[26]}$ Table 1 Thermodynamic data for reversible $\mathrm{H}_{2}$ storage based on methanol $/ \mathrm{CO}_{2}$

\begin{tabular}{|c|c|c|}
\hline No. & Eq. & $\Delta H^{0} /\left(\mathrm{kJ} \cdot \mathrm{mol}^{-1}\right)$ \\
\hline (1) & $\mathrm{CH}_{3} \mathrm{OH}(\mathrm{l}) \rightarrow \mathrm{HCHO}(\mathrm{g})+\mathrm{H}_{2}(\mathrm{~g})$ & +129.8 \\
\hline (2) & $\mathrm{CH}_{3} \mathrm{OH}(\mathrm{g}) \rightarrow \mathrm{CO}(\mathrm{g})+2 \mathrm{H}_{2}(\mathrm{~g})$ & +94.6 \\
\hline (3) & $\mathrm{CH}_{3} \mathrm{OH}(\mathrm{l}) \rightarrow \mathrm{CO}(\mathrm{g})+2 \mathrm{H}_{2}(\mathrm{~g})$ & +127.9 \\
\hline (4) & $\mathrm{CH}_{3} \mathrm{OH}(\mathrm{g})+\mathrm{H}_{2} \mathrm{O}(\mathrm{g}) \rightarrow 3 \mathrm{H}_{2}(\mathrm{~g})+\mathrm{CO}_{2}(\mathrm{~g})$ & +53.3 \\
\hline (5) & $\mathrm{CH}_{3} \mathrm{OH}(\mathrm{l})+\mathrm{H}_{2} \mathrm{O}(\mathrm{l}) \rightarrow 3 \mathrm{H}_{2}(\mathrm{~g})+\mathrm{CO}_{2}(\mathrm{~g})$ & +130.7 \\
\hline
\end{tabular}

目前, 工业上已经实现了使用多相催化剂体系催化 甲醇重整脱氢工艺. 以 $\mathrm{CuO} / \mathrm{ZnO} / \mathrm{Al}_{2} \mathrm{O}_{3}$ 多相催化剂为代
表的铜基催化剂 ${ }^{[27]}$ 已商业化生产，其优点是活性高、选 择性高, 以及成本较低, 但反应需要 $300{ }^{\circ} \mathrm{C}$ 以上的高 温，这会使金属烧结失活并自燃. 以钯-锌合金催化剂 为代表的金属基催化剂 ${ }^{[28]}$ 比铜基催化剂稳定，但是活 性较低. Dumesic 等 ${ }^{[29]}$ 首次报道了使用多相催化剂 $\mathrm{Pt} /$ $\mathrm{Al}_{2} \mathrm{O}_{3}$ 在温度为 $200 \sim 225{ }^{\circ} \mathrm{C}$ 时催化甲醇水相重整. 2017 年, Lin 等 ${ }^{[30]}$ 研发出一种 $\mathrm{Pt} / \alpha-\mathrm{MoC}$ 双功能催化剂, 该催 化剂可以使甲醇水相产氢在 $150 \sim 190{ }^{\circ} \mathrm{C}$ 下发生，且转 化频率达到 $18046 \mathrm{~h}^{-1}, \alpha-\mathrm{MoC}$ 诱导水解离. 多相催化剂 虽然可以催化甲醇大量产氢, 但是催化条件仍需要高 温、高压 ${ }^{[31]}$, 这对甲醇的稳定性是极其不利的, 还可能 产生大量有毒的 $\mathrm{CO}^{[32]}$, 因此不适用于燃料电池, 所以 当下人们想将高温系统转化为低温系统 $\left(<150{ }^{\circ} \mathrm{C}\right)$. 近 年来, 在温和条件下利用均相催化剂催化甲醇脱氢的研 究取得了很大的进展. 本文将主要介绍钽、铑、铱、铁、 锰基均相催化剂在甲醇脱氢反应方面的应用.

\section{1 铂系催化剂}

\section{1 钉基催化剂}

对于甲醇直接脱氢, $\mathrm{Ru}$ 基催化剂是目前研究最广 泛、最成熟的. 相比于其他贵金属而言，钓基催化剂价 格低廉、催化性能高 ${ }^{[33]}$. 早在 1985 年, Maitlis 等 ${ }^{[34]}$ 首次 使用 $\left[\mathrm{Ru}\left(\mathrm{PPh}_{3}\right)_{3} \mathrm{Cl}_{2}\right]$ (1) 催化甲醇直接脱氢合成甲酸甲 酯，这对甲醇脱氢领域的发展具有开创性的历史意义. 在 $150{ }^{\circ} \mathrm{C}$ 时反应 $18 \mathrm{~h}$ 后氢气的转化数为 65 (表 2). 其缺 点是催化剂失活为 $\left[\left(\mathrm{PPh}_{3}\right)_{2} \mathrm{Ru}(\mathrm{CO})(\mu-\mathrm{H})(\mu-\mathrm{Cl})_{2}(\mathrm{CO})-\right.$ $\left(\mathrm{PPh}_{3}\right)_{2}$ ]使反应结束, 另外 $150{ }^{\circ} \mathrm{C}$ 的温度不利于催化剂 的稳定. 在同一年, Saito 等 ${ }^{[35]}$ 证实了由 $\left[\mathrm{Ru}_{2}(\mathrm{OAc})_{4} \mathrm{Cl}\right]$ 和 $\mathrm{PEt}-\mathrm{PPh}_{2}$ 反应生成的 $\left[\mathrm{Ru}(\mathrm{OAc}) \mathrm{Cl}\left(\mathrm{PEtPPh}_{2}\right)_{3}\right]$ (2)可以催 化甲醇直接脱氢为甲醛，该反应无副产物. 在 $66{ }^{\circ} \mathrm{C}$, 加入 2 equiv.乙酸, 反应 $90 \mathrm{~h}$ 后总转化数为 34(表 2), 初 始转化频率为 $0.96 \mathrm{~h}^{-1}$, 平均转化频率为 $0.38 \mathrm{~h}^{-1}$, 如不 加乙酸, 初始转化频率则为 $0.60 \mathrm{~h}^{-1}$. 随后, 该课题 组 ${ }^{\left[{ }^{[3]}\right]}$ 是出了 $\left[\mathrm{Ru}(\mathrm{OAc}) \mathrm{Cl}\left(\mathrm{PEtPPh}_{2}\right)_{3}\right] 2$ 催化甲醇脱氢的反 应机理(Scheme 1). 首先，甲醇与中心金属钉原子结合， 使得乙酸双齿配体变为单齿构型 2a. 随后，甲醇配体上 的氢转移到乙酸配体上, 使得乙酸解离. 然后, 甲氧基 上 $\mathrm{C}-\mathrm{H}$ 键断裂, 氢与中心金属钉结合, 生成钓氢化物 2c. 最后, 钓氢化物与解离的乙酸结合, 生成甲醛并释 放氢气再生初始催化剂. 由此可见, 该反应不会产生一 氧化碳和甲烷等气体污染物, 为后续甲醇脱氢领域向节 能环保方向发展奠定了坚实的基础。尽管 $\left[\mathrm{Ru}(\mathrm{OAc}) \mathrm{Cl}\left(\mathrm{PEtPPh}_{2}\right)_{3}\right] 2$ 能够在较低温度下使甲醇脱 氢, 但其转化效率很低, 反应速率很慢. 
表 2 用于甲醇热脱氢的均相钉催化剂活性

Table 2 Active homogeneous ruthenium catalysts for methanol thermal dehydrogenation.

\begin{tabular}{|c|c|c|c|c|c|c|c|}
\hline Catalyst (mmol) & $\mathrm{MeOH} / \mathrm{mL}$ & Additive (mmol) & $T /{ }^{\circ} \mathrm{C}$ & Time/h & $\mathrm{TOF}^{a} / \mathrm{h}^{-1}$ & $\mathrm{TON}^{b}$ & Ref. \\
\hline$\left[\mathrm{RuCl}_{2}\left(\mathrm{PPh}_{3}\right)_{3}\right](\mathbf{1})(0.08)$ & 10 & - & 150 & 18 & 3.60 & 65 & [34] \\
\hline$\left[\mathrm{Ru}(\mathrm{OAc}) \mathrm{Cl}\left(\mathrm{PEtPPh}_{2}\right)_{3}\right](\mathbf{2})(0.1)$ & 400 & $\mathrm{CH}_{3} \mathrm{COOH}(0.2)$ & 66 & 90 & 0.38 & 34 & [35] \\
\hline$\left[\mathrm{Ru}(\mathrm{H})_{2}\left(\mathrm{~N}_{2}\right)\left(\mathrm{PPh}_{3}\right)_{3}\right](3)\left[(1 \sim 5) \times 10^{-3}\right]$ & 5 & $\mathrm{NaOH}(5)$ & 150 & 2 & 6.40 & 12.8 & [37] \\
\hline$\left[\mathrm{Ru}(\mathrm{H})_{2}\left(\mathrm{PPh}_{3}\right)_{4}\right](4)\left[(1 \sim 5) \times 10^{-3}\right]$ & 5 & $\mathrm{NaOH}(5)$ & 150 & 2 & 7.50 & 15 & [37] \\
\hline $\mathrm{RuCl}_{3} \cdot 3 \mathrm{H}_{2} \mathrm{O}(\mathbf{5})(1)$ & 200 & $\mathrm{MeONa}(610)$ & 79 & n.r..$^{c}$ & $1.68^{d}$ & - & [38] \\
\hline
\end{tabular}

${ }^{a}$ Average TOF referred to overall reaction time. ${ }^{b}$ TON is referred to hydrogen production. ${ }^{c}$ n.r. not reported. ${ }^{d}$ Initial TOF.

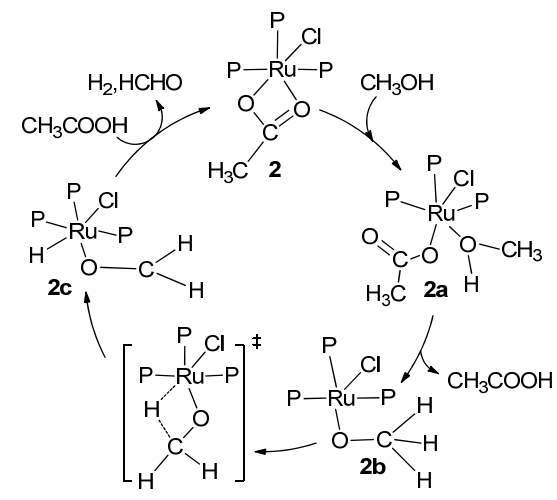

图式 1 甲醇脱氢为甲醛的机理 ${ }^{[36]}$

Scheme 1 Proposed mechanism for the dehydrogenation of methanol to formaldehyde ${ }^{[36]}$

人们致力于开发转化频率高、产氢速率快的催化体

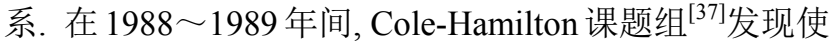
用 $\left[\mathrm{Ru}(\mathrm{H})_{2}\left(\mathrm{~N}_{2}\right)\left(\mathrm{PPh}_{3}\right)_{3}\right](3)$ 或 $\left[\mathrm{Ru}(\mathrm{H})_{2}\left(\mathrm{PPh}_{3}\right)_{4}\right](4)$ 在 $150{ }^{\circ} \mathrm{C}$ 下催化甲醇脱氢时加入十倍的碱可以大幅度提高产氢 速率(表 2). 随后 Saito 等 ${ }^{[38]}$ 发现 $\mathrm{RuCl}_{3} \cdot 3 \mathrm{H}_{2} \mathrm{O} 5$ 不需要辅 助配体也可以催化甲醇脱氢. 在反应体系中加入 $20 \%$ 甲 醇钠, 转化频率由最初无碱时候的 $0.02 \mathrm{~h}^{-1}$ 提高到 1.68 $\mathrm{h}^{-1}$ (表 2), 增加了 84 倍. 2010 年, Nicolas 和 Michael ${ }^{[39]}$ 的理论研究指出碱可以充当一种 “辅助配体”, 促进活 性物种之间的质子转移, 这说明碱在催化甲醇脱氢中起 非常重要的作用. 控制碱的添加量对反应效率有很大的 影响, 反应速率及产量会因碱的添加有所提升, 但高浓 度的碱会使催化剂失活. 从表 2 中我们可以看到, 大多 数早期报道的甲醇脱氢系统的活性和选择性相对较低.

近年来，随着国内外对过渡金属配合物催化甲醇直 接脱氢反应的大量研究, 终于在甲醇水相重整脱氢方面 取得了突破性进展. 2013 年 Grützmacher ${ }^{[40]}$ 和 Beller ${ }^{[4]]}$ 课题组利用甲醇的水相重整克服了一氧化碳使催化剂 中毒的问题, 实现了甲醇的选择性脱氢. Grützmacher 课 题组 ${ }^{[40]}$ 使用催化剂 $6\left(10 \times 10^{-3} \mathrm{mmol}, 0.5 \mathrm{~mol} \%\right)$, 在 $90{ }^{\circ} \mathrm{C}$ 时，加入甲醇 $(2 \mathrm{mmol} 、 81 \mu \mathrm{L})$ 、水 $(2.6 \mathrm{mmol} 、 47$ $\mu \mathrm{L})$ 、苯 $(1 \mathrm{mmol} 、 110 \mu \mathrm{L}$ 作为内标) 和 $\mathrm{THF}(1 \mathrm{~mL})$, 在敞 开系统中, 反应 $10 \mathrm{~h}$ 以上, 转化数 TOF 可达到 540 , 转 化频率 TON 可达到 $54 \mathrm{~h}^{-1}(1 \mathrm{~mol}$ 催化剂催化生成 $1 \mathrm{~mol}$ 氢气为一个转化过程)(表 3). 该反应溶液中没有检测到
甲醛或乙缩酫，这表明甲醇脱氢生成甲醛后水会继续攻 击甲醛，进一步脱氢. 另外催化剂 6 可以在 $90{ }^{\circ} \mathrm{C}$ 催化 甲酸分解, $1 \mathrm{~min}$ 内转化频率可高达 $24000 \mathrm{~h}^{-1}$. 经过一 系列的实验表征以及机理研究，提出了该反应可能的催 化循环, 如 Scheme 2 左环所示. 第一步, 催化剂 6 不与 甲醇直接反应，而是先与水反应得到一个活性适中的钉 化合物 6a，期间会产生 1 分子氢气和碱. 第二步, 底物 分子甲醇与钉化合物 $6 \mathrm{a}$ 中的 $\mathrm{Ru}-\mathrm{N}$ 键结合，从而形成 烯胺基化合物 $6 \mathbf{b}$. 第三步，化合物 $\mathbf{6 b}$ 配体的 $\alpha-\mathrm{C}-\mathrm{H}$ 键 被活化，使得化合物 $\mathbf{6 b}$ 释放出脱氢产物而形成亚胺一氨 基 $\mathrm{Ru}(0)$ 配合物 $\mathbf{6 c}$. 第四步，配合物 $\mathbf{6 c}$ 重复上一步活性 位点的过程形成二氨基 $\mathrm{Ru}(0)$ 配合物 $\mathbf{6 d}$ 并释放出等摩尔 氢气. 第五步，中间体 6d 与碱反应使得初始催化剂 6 再 生，并有等摩尔氢气放出. 其中用 $\mathrm{X}$ 射线表征了生成的 中间体 6d. 化合物 $6 \mathrm{a}$ 和 $6 \mathrm{c}$ 可以催化甲醇、谐二醇或甲 酸脱氢(化合物 6a 可以催化甲酸脱氢释放二氧化碳并形 成化合物 $\mathbf{6 c}$ ). 脱氢过程时配体既具有催化活性也具有 氧化性，可使金属钓的氧化态在 2 和 0 之间变化.
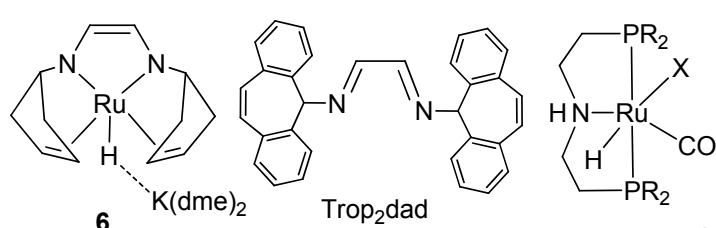

$\mathrm{R}=\mathrm{Ph} \mathrm{X}=\mathrm{Cl} 7$ $\mathrm{R}=i \mathrm{Pr} \mathrm{X}=\mathrm{Cl} 8$ $\mathrm{R}=\mathrm{Ph} \quad \mathrm{X}=\mathrm{BH}_{4} 9 \mathrm{a}$

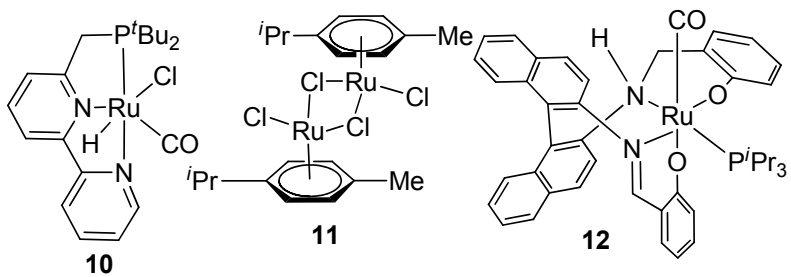

图 2 甲醇脱氢的钉基催化剂

Figure 2 Ru-based catalysts for dehydrogenation of methanol

2015 年, Hall 等 ${ }^{[42]}$ 使用密度泛函理论(DFT)方法报 道了 6 催化甲醇脱氢的另一种反应机制, 如 Scheme 3 所 示. 首先 $\mathrm{Ru}$ 上的氢转移到烯基碳位，随后甲醇不与钉 中心结合直接脱氢，而是与 $\mathrm{N}$ 形成氢键，这是一种基于 配体-配体双功能理论机理, 与 Scheme 2 中 Grützmacher 
表 3 用于甲醇水相重整的均相钉催化剂活性

Table 3 Active homogeneous ruthenium catalysts for methanol reforming

\begin{tabular}{|c|c|c|c|c|c|c|c|c|}
\hline Cat. $\left(10^{-3} \mathrm{mmol} ; 0.001 \%\right.$ to $\left.\mathrm{MeOH}\right)$ & $\mathrm{MeOH} / \mathrm{mr}$ & $\mathrm{I}_{2} \mathrm{O} / \mathrm{mm}$ & 1 Solvent (mL) & dditive (mmol) & $T /{ }^{\circ} \mathrm{C}$ & Time/h & $\mathrm{TOF}^{a} / \mathrm{h}^{-1}$ & ${ }^{1} \mathrm{TON}^{b}$ Ref. \\
\hline $\begin{array}{l}{\left[\mathrm{K}(\mathrm{dme})_{2}\right]\left[\mathrm{RuH}\left(\text { trop }_{2} \mathrm{dad}\right)\right](\mathbf{6})} \\
(10 ; 500)\end{array}$ & 2 & 2.6 & THF (1) & - & 90 & 10 & 54 & $540 \quad[40]$ \\
\hline $\begin{array}{l}{\left[\mathrm{Ru}(\mathrm{H}) \mathrm{Cl}(\mathrm{CO})\left(\mathrm{PNP}-{ }^{i} \mathrm{Pr}\right)\right](\mathbf{8})} \\
(0.88 ; 1)\end{array}$ & 890 & 222 & - & $\mathrm{KOH}(320)$ & 91 & 576 & 613 & $353409[41]$ \\
\hline $\begin{array}{l}{\left[\mathrm{Ru}(\mathrm{H})\left(\mathrm{BH}_{4}\right)(\mathrm{CO})(\mathrm{PNP}-\mathrm{Ph})\right](\mathbf{9 a})} \\
(5 ; 22.5) /\left[\mathrm{Ru}(\mathrm{H})_{2}(\mathrm{dppe})_{2}\right](\mathbf{9 b})(5 ; 22.5)\end{array}$ & 222 & 55.5 & Triglyme (4) & - & 93.5 & 192 & 22 & $4286[50]$ \\
\hline $\begin{array}{l}{\left[\mathrm{Ru}(\mathrm{H}) \mathrm{Cl}(\mathrm{CO})\left(\mathrm{NNP}^{-}{ }^{t} \mathrm{Bu}\right)\right](\mathbf{1 0})} \\
(5 ; 250)\end{array}$ & 20 & 111 & Toluene (2) & $\mathrm{KOH}(40)$ & $100 \sim 105$ & 648 & 44 & $28661[51]$ \\
\hline
\end{tabular}

${ }^{a}$ Average TOF referred to overall reaction time. ${ }^{b}$ TON is referred to hydrogen production. ${ }^{c}$ Initial TOF.

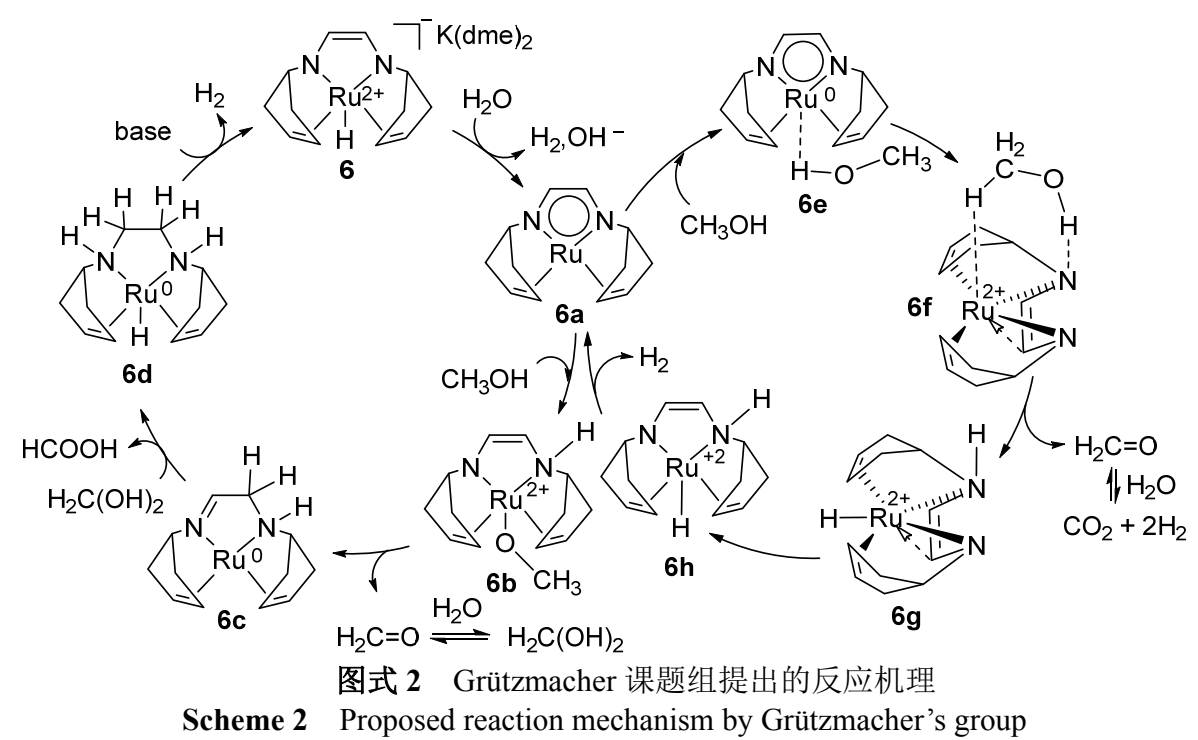

课题组最先提出的甲醇与钉中心原子结合的机理路径 不同, 钓在此过程中只是“旁观者”. 2016 年杨新征等 ${ }^{[43]}$ 使用 DFT 方法研究了 Grützmacher 提出机理的可行性,

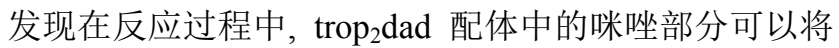
甲醇和水中的氢原子转化为氢气释放出来, 起到储氢的 作用. 此外, 溶剂对该系统的反应能垒有很大的影响, 这说明溶剂有可能改进该系统的反应活性. 2018 年 Grützmacher 课题组 ${ }^{[4]}$ 用 DFT 方法验证了 Ru 中心金属 原子并不仅仅是 “旁观者”, $\mathrm{dad}$ 配体部分与 $\mathrm{Ru}$ 原子共 同作用降低了反应路径过渡态的能垒(Scheme 2, 右环), 这种机理更可行. 该计算使用的模型是真实结构而不是 简化模型，说明简化模型存在一定的非系统误差，应尽 量避免.

基于金属与配体结合的协同催化模式, 钳形 RuPNP 催化剂 7 和 8 被合成并用于催化酯加氢(7) ${ }^{[45]}$ 以及 除甲醇以外的无受体醇脱氢反应 $(8)^{[46]}$, Beller 课题组 ${ }^{[41]}$ 发现这两种钓配合物也可以催化甲醇水相重整, 但需要 在碱性条件下进行. 使用 $49.3 \mu \mathrm{mol} 、 250 \mathrm{mg} / \mathrm{L}$ 催化剂 7, 在 $72{ }^{\circ} \mathrm{C}$ 下, 加入 $8 \mathrm{~mL} \mathrm{MeOH} 、 2 \mathrm{~mL} \mathrm{H}_{2} \mathrm{O} 、 0.1 \mathrm{~mol} / \mathrm{L}$ $\mathrm{NaOH}$ ，催化甲醇脱氢可以释放出 $\mathrm{H}_{2}$ 和 $\mathrm{CO}_{2}$. 使用 1.58

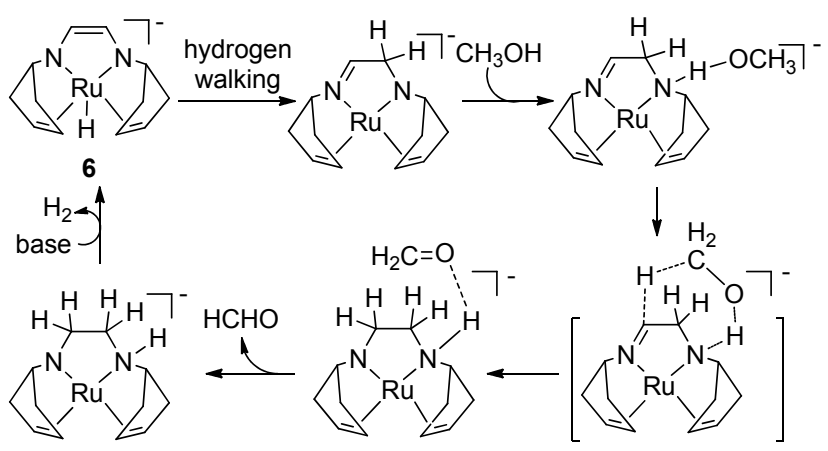

图式 3 Hall 等提出的反应机理

Scheme 3 Proposed reaction mechanism by Hall and co-workers

$\mu \mathrm{mol} 、 6.4 \mathrm{mg} / \mathrm{L}$ 催化剂 8 , 在 $95{ }^{\circ} \mathrm{C}$ 下, 加入 $10 \mathrm{~mL}$ 甲醇、 $8 \mathrm{~mol} / \mathrm{L} \mathrm{KOH}$, 反应 $3 \mathrm{~h}$ 后, 催化甲醇脱氢的平均转化频 率可高达 $4723 \mathrm{~h}^{-1}$. 由于该反应放出的 $\mathrm{CO}_{2}$ 会被碳酸盐 吸收, 且几乎不产生 $\mathrm{CO}$ 污染物, 所以可以得到很纯的 氢气. 催化剂 8 连续催化甲醇脱氢反应 3 个星期之后, 氢气的转化数可高达 350000 , 在最后的 $24 \mathrm{~h}$ 测得其平 均转化频率为 $200 \mathrm{~h}^{-1}$ (表 3).

随着碱的消耗和沸点的降低, 催化剂活性逐渐下 
降. 由此看出, 催化剂 8 只是一个催化前体, 雷鸣 ${ }^{[47}$ 、 Beller $^{[48]}$ 、杨新征 ${ }^{[49]}$ 等课题组的密度泛函理论研究表明, 与等摩尔的碱反应才可以使其转化为具有催化活性的 酰胺配合物 8a. 然后甲醇羟基上的氢与酰胺配合物 $8 \mathbf{a}$ 上的 $\mathrm{N}$ 结合, $\alpha-\mathrm{C}-\mathrm{H}$ 键上的氢与中心金属钉原子结合 使得反应生成二氢化物 8b (Scheme 4). 由此可见, 酰胺 配合物 $8 \mathrm{a}$ 和二氢化物 $8 \mathrm{~b}$ 是甲醇、甲酸和谐二醇脱氢的 关键化合物 ${ }^{[41]}$.

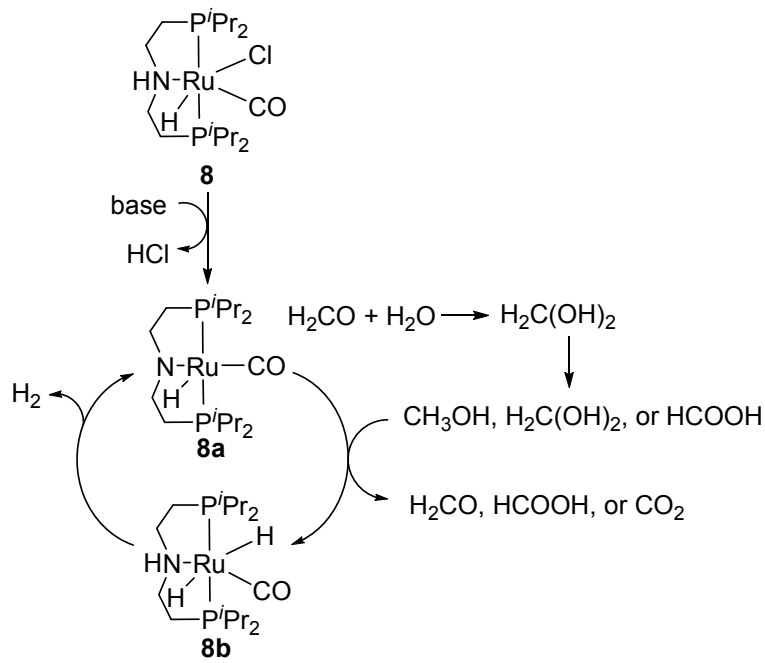

图式 $4\left[\mathrm{Ru}(\mathrm{H}) \mathrm{Cl}(\mathrm{CO})\left(\mathrm{PNP}-{ }^{i} \mathrm{Pr}\right)\right](8)$ 催化甲醇水相脱氢的简化 循环机理

Scheme 4 Simplified catalytic cycle for the aqueous dehydrogenation of $\mathrm{MeOH}$ catalyzed by $\left[\mathrm{Ru}(\mathrm{H}) \mathrm{Cl}(\mathrm{CO})\left(\mathrm{PNP}-{ }^{i} \mathrm{Pr}\right)\right](\mathbf{8})$

使用催化剂 7 和 8 进行甲醇脱氢的最大缺点是都需 要高浓度的碱. 由于在溶液中检测到了游离的甲酸盐, 所以高浓度的碱有可能减慢甲醇产氢速率. 因此可以设 想, 加大催化剂的用量, 使用对甲酸分解有效的第二种 催化剂 8 或是高沸点溶剂就可以在无碱条件下进行甲醇 脱氢. Beller 等 ${ }^{[50]}$ 经过对催化剂和溶剂的篎选，报道了 一种双催化系统, Ru-MACHO- $\mathrm{BH}_{4}$ (9a) 和 $\left[\mathrm{Ru}(\mathrm{H})_{2}-\right.$ $\left.(\text { dppe })_{2}\right]($ dppe $=1,2-$ bis (diphenylphosphino)ethane) $(9 b)$ 在 三甘醇二甲醚溶液中共同作用可以不需要碱去激活活 性位点来催化甲醇脱氢. 如果两种催化剂单独催化甲醇 脱氢, 活性极低, 但是当它们共同加入反应体系时, 甲 醇脱氢产率迅速提高. 利用 $5 \mu \mathrm{mol}$ 催化剂 $9 \mathrm{a}$ 和 $5 \mu \mathrm{mol}$ 催化剂 9b, 在 $93.5{ }^{\circ} \mathrm{C}$ 时, 加入 $9 \mathrm{~mL} \mathrm{MeOH} 、 1 \mathrm{~mL} \mathrm{H}_{2} \mathrm{O}$ 、 $4 \mathrm{~mL}$ 三甘醇二甲醚, 催化甲醇脱氢反应 $7 \mathrm{~h}$ 后, 转化频 率为 $93 \mathrm{~h}^{-1}, 8 \mathrm{~d}$ 之后反应结束, 总转化数为 4286(表 3).

在 2014 年, Milstein 课题组 ${ }^{[51]}$ 证实一种带有 PNN 钳 形配体的钉催化剂 10 可以催化甲醇水蒸气制氢. 在 $100{ }^{\circ} \mathrm{C}$ 下, 加入 $2 \mathrm{~mol} \mathrm{KOH}$, 使用 $0.025 \mathrm{~mol} \%$ 催化剂 $\mathbf{1 0}$ 催化甲醇脱氢反应 $7 \mathrm{~d}$ 后，氢气产率可达 $70 \% ; 9 \mathrm{~d}$ 后， 产率升到 $90 \%$, 平均转化频率分别为 50 和 $45 \mathrm{~h}^{-1}$ (表
$3)^{[51]}$. 上述反应在有机溶液中才可以检测到催化活性, 所以 $2 \mathrm{~mL}$ 甲苯的加入是用来增加催化剂的溶解性. 该 催化剂不需要离析和纯化就可以重复多次使用, 寿命长 达一个多月。要想让甲醇脱氢生成甲酸中间体，就必须 使催化剂 10 在碱性环境下生成具有真正催化活性的物 种 $10 \mathrm{a}$, 化合物 $10 \mathrm{a}$ 与底物分子反应，使得金属钉原子 的另一侧上也有氢原子，从而得到钌二氢化物 $10 \mathrm{~b}$, 同 时释放出氢气(Scheme 5).

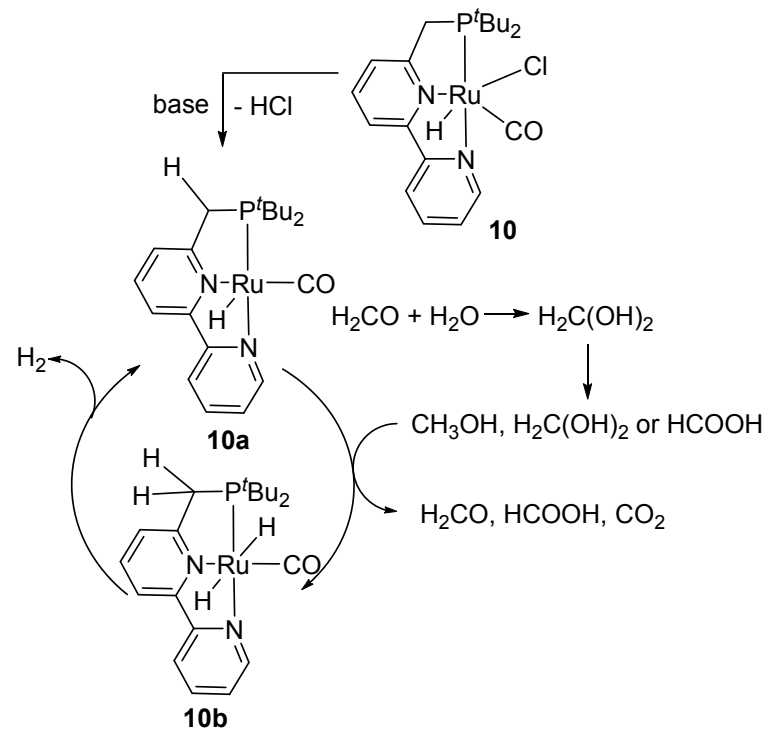

图式 5 络合物 $\mathbf{1 0}$ 催化甲醇脱氢的关键中间体 ${ }^{[14,52]}$

Scheme 5 Possible key-intermediates in the aqueous dehydrogenation of $\mathrm{MeOH}$ catalyzed by complex $\mathbf{1 0}$

上述三类催化剂(图 3)的共同特点是具有多齿配体, 这使得金属配合物的热稳定性有了显著提高. 多齿配体 可能具有阻挡金属活性位点的作用，防止甲醇脱氢后甲 醛进一步脱羰，这对于甲醇脱氢的选择性是非常重要 的. 总的来说，它们的反应机制为金属与配体协同作用， 如 Scheme 6 所示，主要包括以下四步：(1)甲醇脱氢生成 甲醛和氢气; (2)甲醛与水生成谐二醇 ${ }^{[52]} ;(3)$ 谐二醇脱氢 为甲酸同时释放氢气; (4)甲酸脱羧分解为二氧化碳和氢 气，这些参与氧化还原反应的多功能配体 ${ }^{[53]}$ 被称为 “非 单一(non-innocent)” 协同配体 ${ }^{[54 a]}$.

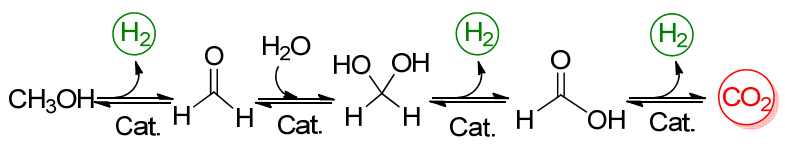

图式 6 均相催化甲醇水相重整过程

Scheme 6 Crucial steps in the homogeneously catalyzed aqueous-phase methanol reforming

通过比较甲醇热分解(表 2)和甲醇水相重整脱氢(表 3)数据，可以得出带有 “非单一” 配体的钉催化剂远比 没有辅助配体的钓催化剂活性高. 这可能是因为前者中 
催化剂与底物结合或是 $\mathrm{C}-\mathrm{H}$ 键活化只发生在金属中 心，需要更高的活化能，而后者采用的是金属与配体协 同作用的反应机制, 反应活化能垒较低, 使得反应更容 易进行, 从而活性相对更高 ${ }^{[54 b]}$. 随着甲醇脱氢均相催 化剂在 $60 \sim 100{ }^{\circ} \mathrm{C}$ 范围内的发展, 生物酶催化体系也 得到了探索, 其目的是实现室温下甲醇水相重整脱 氢 ${ }^{[55]}$. 在 2015 年, Prechtl 等 ${ }^{[56]}$ 报道了一种室温下生物诱 导甲醇水相脱氢的方法. 这种方法是利用 $\left[\mathrm{RuCl}_{2}(p-\right.$ cymene) $]_{2} 11$ 与氧化酶和过氧化氢酶共同作用, 在碳酸 盐的缓冲溶液中以 $3.2 \mu \mathrm{mol} / \mathrm{mL}$ 的速率进行甲醇脱氢. 酶首先催化甲醇生成二甲醇, 然后利用催化剂 11 进一 步脱氢, 这是反应能在室温下进行的关键.

2016 年 Olah 和 Prakash 等 ${ }^{[57]}$ 阐述了一种甲醇脱氢 的新路径, 即利用二级胺与甲醇反应释放出氢气, 二级 胺可以吸收甲醇脱氢的副产物二氧化碳, 然后还会生成 $N$-甲酰胺和 $N, N^{\prime}$-二甲酰胺, 这些产物也可以可逆加氢 为甲醇和二级胺. 这个反应使用的催化剂是 Ru-PNP 8 , 该催化剂既可以用于脱氢反应也可以用于加氢反应. 在 $120{ }^{\circ} \mathrm{C}$ 下, 使 $N, N^{\prime}$-二甲基乙二胺和甲醇以体积比为 1 : 4 的比例混合, 加入 $5 \mathrm{~mol} \%$ 添加剂 $\mathrm{K}_{3} \mathrm{PO}_{4}$, 并将甲苯作 为溶剂, 利用 $1 \mathrm{~mol} \%$ 催化剂 8 , 催化脱氢反应 $24 \mathrm{~h}$ 后, 得到氢气的产率为 $90 \%$ (Eq. 6). 仅给系统加压到 $40 \mathrm{bar}$ 就可以使其进行逆反应, 得到 $92 \%$ 的 $N, N^{\prime}$-二甲基乙二 胺. 此系统可以避免产生 $\mathrm{CO}_{2}$, 所以不需要再加气体提 纯过程就可以应用于氢气燃料电池.

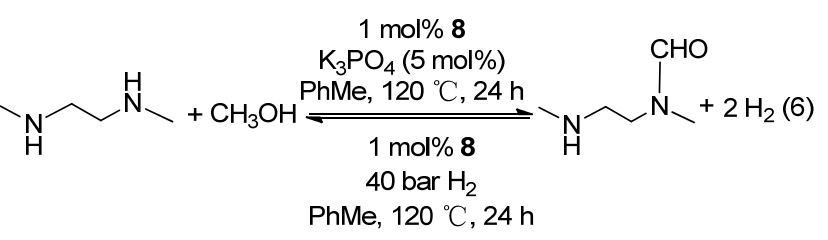

2016 年 Reek 课题组 ${ }^{[58]}$ 利用 $\mathrm{Ru}$ (salbinapht)(CO)(P$\left.{ }^{i} \mathrm{Pr}_{3}\right)$ [salbinapht $=(R)-2$ '2'-bis(salicylideneamino)-1,1'binaphtyl] 12 催化 75\%甲醇和水(其中醇水体积比为 9: 1)与 $25 \% 1,4$-二氧己环反应, 加入 $8 \mathrm{~mol} / \mathrm{L} \mathrm{KOH}$, 在 $82{ }^{\circ} \mathrm{C}$ 时可以得到的最高转化频率为 $55 \mathrm{~h}^{-1}$. 该系统除 了释放出氢气, 还会生成甲酸盐和碳酸盐. 催化剂上的 羰基配体会被亲核试剂 $\left(\mathrm{KOH}\right.$ 或 $\left.\mathrm{H}_{2} \mathrm{O}\right)$ 进攻生成甲酸盐 和氢气. 作者猜想此羰基络合物 12 仅是一种催化前体, 在强碱环境中才可以形成真正具有催化活性的物种. 在 催化反应之后检测出微量的同样具有催化甲醇脱氢活 性的 $\left[\mathrm{RuH}_{2}(\mathrm{CO})_{2}\left(\mathrm{P}-i \mathrm{Pr}_{3}\right)_{2}\right](\mathbf{1 2 a})\left(\mathrm{TOF}=50 \mathrm{~h}^{-1}\right)$.

除以上单核 $\mathrm{Ru}$-基催化剂以外, Shinoda 和 Yamakawa 等 ${ }^{[59]}$ 报道了两种双金属催化体系. 他们利用 $\mathrm{Ru}(\mathrm{II})-\mathrm{Sn}(\mathrm{II})$ 双金属体系 $\left[\mathrm{Ru}\left(\mathrm{SnCl}_{3}\right)_{5}\left(\mathrm{PPh}_{3}\right)\right]^{3-} 13$ 成功 催化甲醇脱氢为乙酸甲酯. 此反应是在 $65{ }^{\circ} \mathrm{C}$ 下 $\mathrm{N}_{2}$ 环境
下进行的, $\mathrm{MeOH}$ 和 $\mathrm{MeNO}_{2}$ 以 $1: 1$ 等体积混合, 反应 $100 \mathrm{~h}$ 后的转化数为 15.7 . 虽然活性低, 但是该反应不需 要任何添加剂, 催化成本较低. 随后该课题组对此反应 的机理研究表明, 第一步甲醇脱氢生成甲醛是决速步 骤, 此外, 有可能是 $\mathrm{Ru}(\mathrm{II})-\mathrm{Sn}(\mathrm{II})$ 双金属系统使得甲酸 甲酯异构化为乙酸 ${ }^{[60]}$. 另一种双金属系统是利用 $\left[\mathrm{CpRu}\left(\mathrm{PPh}_{3}\right)_{2}\left(\mathrm{SnF}_{3}\right)\right]\left(\mathrm{Cp}=\eta^{5}-\mathrm{C}_{5} \mathrm{H}_{5}\right) \quad \mathbf{1 4}$, 在 $140{ }^{\circ} \mathrm{C}$ $\mathrm{MeOH}$ 和 $\mathrm{MeNO}_{2}$ 以 $1: 1$ 体积混合的溶液中, 反应 $40 \mathrm{~h}$ 后转化数可达 $120^{[61]}$. 含氟配体比含氯和溴配体的活性 更高, 原因在于 $\mathrm{Sn}-\mathrm{F}$ 键高电负性增加了 $\mathrm{Ru}$ 带正电荷 的能力, $\mathrm{F}$ 与甲醇的 $\beta-\mathrm{H}$ 易形成氢键, 有利于 $\mathrm{C}-\mathrm{H}$ 键的 解离.

\section{2 铱基催化剂}

近年来，铱基络合物由于在甲醇脱氢方面具有高活 性与高选择性, 受到了人们的关注. 2015 年, Crabtree 等 ${ }^{[2]}$ 报道了一系列能稳定催化甲醇脱氢的双氮杂环羰 基铱催化剂 15, 16 和 17. 因为这三种铱催化剂 $\mathrm{N}$ 杂环上 的取代基不同，所以催化活性也不同，它们的催化活性 以 $\mathrm{R}=\mathrm{Me}>\mathrm{Et}>{ }^{n} \mathrm{Bu}$ 的顺序降低. 使用 $0.0013 \mathrm{~mol} \%$ 催 化剂 15 , 在 $91{ }^{\circ} \mathrm{C}$ 时, 加入 $6.7 \mathrm{~mol} / \mathrm{L} \mathrm{KOH}$ 溶液和 $3 \mathrm{ml}$ 甲醇(其中甲醇既是反应物又是溶剂), 反应 $40 \mathrm{~h}$ 后, 甲 醇脱氢的转化数可以高达 $8000^{[63]}$. 而且, 在该反应中, 几乎不产生 CO.

在同一年, Yamaguchi 等 ${ }^{[64]}$ 成功实现了利用铱催化 剂从甲醇水溶液中脱氢. 他们使用的是带双功能吡啶酮 盐配体的阴离子铱配合物 18 作为催化剂. 相比于之前 的反应体系, 这个系统只需要加入 $0.046 \mathrm{~mol} / \mathrm{L} \mathrm{NaOH}$, 甲醇和水体积比为 $1: 4$, 反应 $15 \mathrm{~h}$ 后, 产氢率可达 $64 \%$, 转化数为 10510 . 需要注意的是, 该反应 $\mathrm{pH}$ 值需 要控制在 $8 \sim 12$ 之间, 以确保阴离子催化剂的稳定性, 在 $\mathrm{pH}$ 值较低的情况下, 阴离子转变为中性离子和阳离 子, 使得催化剂失活. 在类似的条件下, 反应得到的甲 醛和甲酸钠作为底物也可以大量产氢.
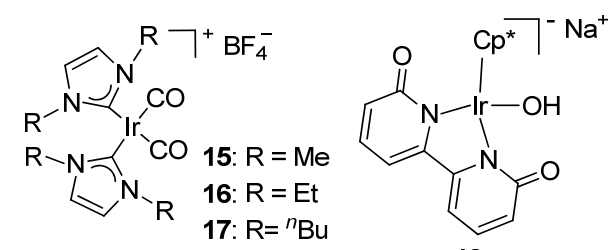

18
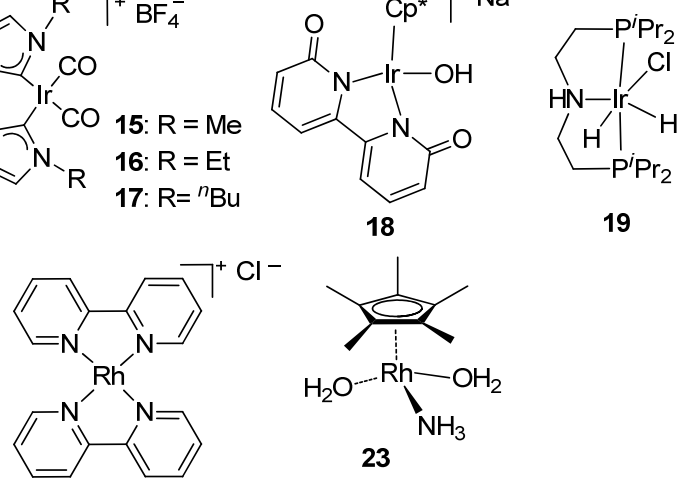

22

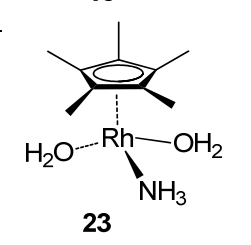

19 
在 2017 年, Beller 课题组 ${ }^{[65]}$ 报道了一种 Ir-PNP 络合 物 19 可以使甲醇水相重整脱氢.与 Ru-PNP 和 Fe-PNP 体系相比, 配合物 19 在低浓度碱中活性更高, 高浓度碱 中稳定性更好. 使用 Ir-PNP 催化剂, 加入 $0.5 \mathrm{~mol} / \mathrm{L}$ $\mathrm{KOH}$ 以及 $10 \mathrm{~mL}$ 体积比为 $9: 1$ 的 $\mathrm{MeOH}$ 和水, 在 $70{ }^{\circ} \mathrm{C}$ 时, 反应 $1 \mathrm{~h}$ 后 $\mathrm{TOF}$ 为 $326 \mathrm{~h}^{-1}$, 反应可持续 $16 \mathrm{~h}$, TON 为 1400 . 把 $\mathrm{KOH}$ 的浓度降到 0.1 , 反应 $1 \mathrm{~h}$ 后的 $\mathrm{TOF}$ 为 $525 \mathrm{~h}^{-1}$; 把 $\mathrm{KOH}$ 的浓度升高到 $8.0 \mathrm{~mol} / \mathrm{L}$, 反应 可持续 $60 \mathrm{~h}, \mathrm{TON}$ 为 1900 . 由此可看出, 碱浓度对催化 剂活性和稳定性均有显著影响.

基于生物诱导方法, 2017 年周小春等 ${ }^{[66]}$ 报道了铱催 化剂和酶诱导催化体系可以共同作用促使甲醇重整脱 氢. 该系统主要包括两个过程: $\mathrm{CH}_{3} \mathrm{OH} \rightarrow \mathrm{HCOOH} \rightarrow \mathrm{H}_{2}$ 和 $\mathrm{CH}_{3} \mathrm{OH} \rightarrow \mathrm{NADH} \rightarrow \mathrm{H}_{2}$. 先使用脱氢酶将甲醇同时氧 化为甲酸和 $\mathrm{NADH}$ ，随后利用催化剂 $\left[\mathrm{Cp}^{*} \mathrm{IrCl}_{2}\right.$ (ppy)] (ppy=聚吡咯) 20 和 $[\mathrm{Cp} * \operatorname{IrCl}(\mathrm{phen})] \mathrm{Cl} 21$ 催化甲酸和 $\mathrm{NADH}$ 释放出氢气(Scheme 7). 该反应可使 $400 \mathrm{mmol} / \mathrm{L}$ 甲醇溶液 $(\mathrm{pH}=8.05)$ 在 $30{ }^{\circ} \mathrm{C}$ 时反应 $32 \mathrm{~h}$ 后生成 $4 \mu \mathrm{mol}$ 氢气.

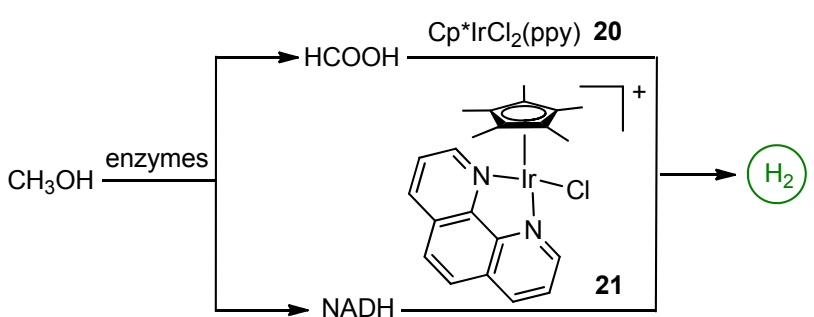

图式 7 酶和铱催化剂共同作用的生物诱导甲醇重整方法 Scheme 7 Bioinduced approach for methanol reforming based on a combination of enzymes and iridium catalysts

\section{3 铑基催化剂}

1987 年, Cole-Hamilton 课题组 ${ }^{[67]}$ 使用 $\left[\mathrm{Rh}(\text { bipy })_{2}\right] \mathrm{Cl}$ (bipy $=2,2^{\prime}$-联吡啶) 22 最先成功催化甲醇与水反应生成 二氧化碳和氢气(表 1 中 Eq. 4). 由于这条反应路线吸收 热量较少，使得反应更容易进行. 利用 $5 \times 10^{-6} \mathrm{~mol}$ $\left[\mathrm{Rh}(\text { bipy })_{2}\right] \mathrm{Cl}$, 在 $120{ }^{\circ} \mathrm{C}$ 以及体积比为 $95: 5$ 的醇/水混 合溶液中, 再加 $0.25 \sim 5 \times 10^{-3} \mathrm{~mol}$ 氢氧化钠, 在封闭系 统中反应 $3 \mathrm{~h}$ 后得到的转化频率为 $7 \mathrm{~h}^{-1}$. 当时作者认为 加水在热力学上更有利于醇脱氢, 然而直到 25 年后才 有了水相醇脱氢的突破. 在上述催化剂报道之后, 仅出 现了一种铑基催化剂催化甲醇重整脱氢. 2017 年周小春 等 ${ }^{[68]}$ 利用均相催化剂 $\left[\mathrm{Cp} * \mathrm{Rh}\left(\mathrm{NH}_{3}\right)\left(\mathrm{H}_{2} \mathrm{O}\right)_{2}\right]^{3+} \mathbf{2 3}$ 在 $70{ }^{\circ} \mathrm{C}$ 下脱氢, 其转化频率可达 $83 \mathrm{~h}^{-1}$. 该反应不会产生 使催化剂失活的 $\mathrm{CO}$, 并且将反应溶液的 $\mathrm{pH}$ 值控制在 6 左右催化剂活性最高. 该催化剂进一步推动了低温甲醇 脱氢领域的发展.

\section{2 铁系和其他催化剂}

除了可以用贵金属钓、铑、铱催化剂催化甲醇脱氢, 诸如铁、锰这样地表产量丰富、价格低廉、毒性小或无 毒性的过渡金属同样可以用于甲醇脱氢 ${ }^{[69]}$. 在 2013 年, Beller 等 ${ }^{[70]}$ 首次报道了低温下使用铁螯合配体化合物 $\mathbf{2 4}$ 催化甲醇产氢. 使用催化剂 24, 体积比为 $9: 1$ 的 $\mathrm{MeOH}$ 和 $\mathrm{H}_{2} \mathrm{O}$ 混合溶液中加入 $8 \mathrm{~mol} / \mathrm{L} \mathrm{KOH}$, 在 $91{ }^{\circ} \mathrm{C}$, 第 1 个小时内, 转化频率为 $702 \mathrm{~h}^{-1}, 43 \mathrm{~h}$ 后总转化数为 6270 . 若加入过量的 $\mathrm{HN}\left(\mathrm{CH}_{2} \mathrm{CH}_{2} \mathrm{P}-i \mathrm{Pr}_{2}\right)_{2}$ 配体可以进一步增强 催化剂的稳定性, 延长催化剂的使用寿命, 使得转化数 可以达到 9184 (111 h). 2015 年, Holthausen 等 ${ }^{[71]}$ 报道了 另外几种铁螯合物 25、26、27 和 28. 在 $4: 1 \mathrm{MeOH}$ 和 $\mathrm{H}_{2} \mathrm{O}$ 混合溶液中加入 $\mathrm{LiBF}_{4}$, 使用催化剂 27 在无碱条件 下催化甲醇脱氢, 经过回流, 反应 $94 \mathrm{~h}$ 后转化数可达 51000 , 这是迄今为止第一过渡系金属均相催化甲醇重 整所达到的最高产量.
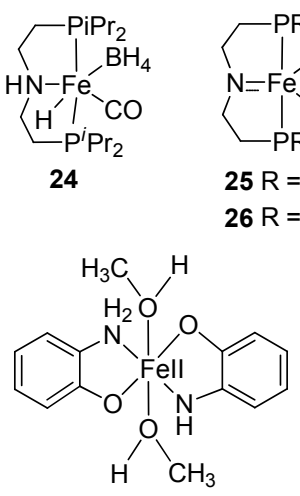

29
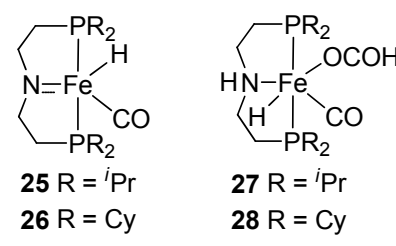

$27 \mathrm{R}={ }^{i} \mathrm{Pr}$ $28 \mathrm{R}=\mathrm{Cy}$

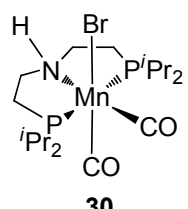

30
2016 年 Chang 等 ${ }^{[72]}$ 使用 $\operatorname{tran}-\left[\mathrm{Fe}^{\mathrm{II}}(\mathrm{apH})_{2}(\mathrm{MeOH})_{2}\right]$ ( $\mathrm{apH}=o$-aminophenolato ligand) 29 光催化剂在室温下实 现了催化无水甲醇重整的反应, 此反应并不需要添加任 何光敏剂就可以释放出氢气并生成甲醛产物.

虽然铁基催化剂成本低、产量高, 但是活性不如钉 基催化剂好. 然而如果将 Crabtree 等 ${ }^{[73]}$ 提出的每单位转 化数成本作为未来绿色催化剂的评判标准，那么铁基催 化剂就会成为首选. 这激励人们对其它第一过渡系金属 催化剂进行研究. Beller 课题组 ${ }^{[74]}$ 报道了首例锰催化剂 30 在甲醇水相脱氢体系中的应用. 虽然其单独使用活 性比不上铁基催化剂, 但在加入过量 $\mathrm{HN}\left(\mathrm{CH}_{2} \mathrm{CH}_{2} \mathrm{P}-\right.$ $\left.i \mathrm{Pr}_{2}\right)_{2}$ 后, 脂肪族锰配合物 $\mathbf{3 0}$ 的稳定性会更好, 可以持 续一个月之久, 并且实现了高达 20000 的转化数. 2018 年, 焦海军课题组 ${ }^{[75]}$ 关于配合物 $\mathbf{3 0}$ 催化甲醇水相脱氢 反应的机理研究表明在强碱条件下需要克服的反应能 垒低于无碱条件，这又一次说明碱在甲醇脱氢反应中的 促进作用. 此外, 该催化剂也可以用于催化乙醇、多聚 甲醛和甲酸脱氢. 


\section{3 结论与展望}

为了满足全球能源需求的不断增长, 迫切需要加快 甲醇脱氢催化剂的发展和应用研究进程. 本文着重从多 种过渡金属催化剂的结构、反应条件、产氢转化频率、 反应机理等方面进行了综述. 最初甲醇热脱氢使用的钉 基催化剂是经典络合物, 为后续一系列研究奠定了基 础. 带有 “非单一” 辅助配体催化剂使得甲醇脱氢在产 量和活性上有了大大的提高. 然而, 这类反应通常需要 高浓度的强碱来激活催化剂的活性位点, 从而有效地释 放氢气, 随着反应的进行会有固体沉淀, 这就需要重新 不断地添加碱, 因而使得产氢效率有所限制, 所以下一 步需要重点研究的方向是不加入任何添加剂直接脱氢. 低温均相过渡金属催化甲醇水相重整是相对较有前景 的领域, 该领域的开创性工作仅开展了 5 年左右, 虽然 报道的最高转化数超过了 300000 , 但仍需要更有效、稳 定、便宜的金属催化剂来使这项工艺最终应用在工业上. 目前, 醇脱氢工作研究的方向已经慢慢从之前的贵金属 钌、铑、铱向相对便宜的铁、锰等金属转变, 虽然活性 不如贵金属, 但是产量更高、成本更低、更为绿色环保. 尽管用于甲醇脱氢的均相催化剂的活性和产率还远远 达不到实际应用的要求, 但很明显这些年关于这一领域 的均相催化剂增加很多. 经过研究工作者们的努力, 相 信在不久的未来终会取得更大的进步.

\section{References}

[1] Armaroli, N.; Balzani, V. Chem. Asian J. 2011, 6, 768.

[2] Behrendt, F.; Schüth, F. Chem. Ing. Tech. 2011, 83, 1984.

[3] Cook, T. R.; Dogutan, D. K.; Reece, S. Y.; Surendranath, Y.; Teets, T. S.; Nocera, D. G. Chem. Rev. 2010, 110, 6474.

[4] Armaroli, N.; Balzani, V. ChemSusChem 2011, 4, 21.

[5] Zheng, J. Y.; Liu, X. X.; Xu, P.; Liu, P. F.; Zhao, Y. Z.; Yang, J. Int. J. Hydrogen Energy 2012, 37, 1048.

[6] Xu, Y.; Tao, Z.-L.; Chen, J. Prog. Chem. 2006, 18, 200 (in Chinese).

(许炜, 陶占良, 陈军, 化学进展, 2006, 18, 200.)

[7] Dalebrook, A. F.; Gan, W.; Grasemann, M.; Moret, S.; Laurenczy, G. Chem. Commun. 2013, 49, 8735.

[8] Chen, J.; Chen, Q.-X.; Chen, Y.-W.; Fan, S.-S.; Wang, Y.-H.; Yang, L.; Lang, X.-M.; Zhang, W.-X.; Huang, Y.; Xiong, W.-T. Energy Storage Sci. Tech. 2015, 4, 131 (in Chinese). (陈俊, 陈秋雄, 陈运文, 樊栓狮, 王燕鸿, 杨亮, 郎雪梅, 张雯 翔, 黄怡, 熊文涛, 储能科学与技术, 2015, 4, 131.)

[9] Chen, Z.; Yang, Y.-Q.; Bao, J.-G.; Wang, W.-Y.; Jiang, X.-M., Chem. Ind. Eng. Prog. 2010, 29, 484 (in Chinese).

(陈卓，杨运泉，包建国，王威燕; 蒋新民，化工进展，2010，29， 484.)

[10] Li, L.-L.; Fan, S.-S.; Chen, Q.-X.; Yang, G.; Wen, Y.-G. Energy Storage Sci. Tech. 2018, 7, 586 (in Chinese). (李璐伶; 柇栓狮; 陈秋雄; 杨光; 温永刚, 储能科学与技术, 2018, 7, 586.)

[11] Niaz, S.; Manzoor, T.; Pandith, A. H. Renew. Sust. Energ. Rev. 2015, 50, 457

[12] Ares, J. R. Int. J. Hydrogen Energy 2014, 39, 9824.

[13] Sordakis, K.; Tang, C.; Vogt, L. K.; Junge, H.; Dyson, P. J.; Beller, M.; Laurenczy, G. Chem. Rev. 2018, 118, 372.
[14] Alberico, E.; Nielsen, M. Chem. Commun. (Camb.) 2015, 51, 6714.

[15] He, T.; Pachfule, P.; Wu, H.; Xu, Q.; Chen, P. Nat. Rev. Mater. 2016, $1,16059$.

[16] Li, H.-W.; Yan, Y.; Orimo, S.-I.; Züttel, A.; Jensen, C. M. Energies 2011, 4, 185 .

[17] Peng, B.; Chen, J. Energ. Environ. Sci. 2008, 1, 479.

[18] Zhu, Q.-L.; Xu, Q. Energ. Environ. Sci. 2015, 8, 478

[19] Klerke, A.; Christensen, C. H.; Nørskov, J. K.; Vegge, T. J. Mater. Chem. 2008, 18, 2304.

[20] Moury, R.; Moussa, G.; Demirci, U. B.; Hannauer, J.; Bernard, S.; Petit, E.; van der Lee, A.; Miele, P. Phys. Chem. Chem. Phys. 2012, $14,1768$.

[21] Zhao, H. Y.; Oyama, S. T.; Naeemi, E. D. Catal. Today 2010, 149, 172.

[22] (a) Grasemann, M.; Laurenczy, G. Energ. Environ. Sci. 2012, 5, 8171.

(b) Mellmann, D.; Sponholz, P.; Junge, H.; Beller, M. Chem. Soc. Rev. 2016, 45, 3954.

[23] Johnson, T. C.; Morris, D. J.; Wills, M. Chem. Soc. Rev. 2010, 39, 81.

[24] Asinger, F. Methanol—Chemie und Energierohstoff, Springer, Berlin, Heidelberg, 1986, pp. 1 9.

[25] Smith, T. A.; Maitlis, P. M. J. Organomet. Chem. 1984, 269, c7.

[26] Wang, W.-H.; Himeda, Y.; Muckerman, J. T.; Manbeck, G. F.; Fujita, E. Chem. Rev. 2015, 115, 12936.

[27] Sá, S.; Silva, H.; Brandão, L.; Sousa, J. M.; Mendes, A. Appl. Catal. B-Environ. 2010, 99, 43.

[28] Iulianelli, A.; Ribeirinha, P.; Mendes, A.; Basile, A. Renew. Sust. Energ. Rev. 2014, 29, 355.

[29] Cortright, R. D.; Davda, R. R.; Dumesic, J. A. Nature 2002, 418, 964.

[30] Lin, L.; Zhou, W.; Gao, R.; Yao, S.; Zhang, X.; Xu, W.; Zheng, S.; Jiang, Z.; Yu, Q.; Li, Y.-W.; Shi, C.; Wen, X.-D.; Ma, D. Nature 2017, $544,80$.

[31] Palo, D. R.; Dagle, R. A.; Holladay, J. D. Chem. Rev. 2007, 107, 3992.

[32] Navarro, R. M.; Peña, M. A.; Fierro, J. L. G. Chem. Rev. 2007, 107, 3952.

[33] Wu, S.; Xiong.X.-D.; Wang, S.-G. Rare Metals 2007, 31, 237 (in Chinese). (吴松; 熊晓东; 王胜国，稀有金属, 2007, 31, 237.)

[34] Smith, T. A.; Aplin, R. P.; Maitlis, P. M. J. Organomet. Chem. 1985, $291, \mathrm{c} 13$.

[35] Shinoda, S.; Itagaki, H.; Saito, Y. J. Chem. Soc., Chem. Commun. $1985,13,860$

[36] Itagaki, H.; Shinoda, S.; Saito, Y., Bull. Chem. Soc. Jpn. 1988, 61, 2291.

[37] Morton, D.; Cole-Hamilton, D. J. J. Chem. Soc. Chem. Commun., 1988, 1154

[38] Fujii, T.; Saito, Y. J. Mol. Catal. 1991, 67, 185

[39] Sieffert, N.; Bühl, M. J. Am. Chem. Soc. 2010, 132, 8056.

[40] Rodríguezlugo, R. E.; Trincado, M.; Vogt, M.; Tewes, F.; Santisoquinones, G.; Grützmacher, H. Nat. Chem. 2013, 5, 342.

[41] Nielsen, M.; Alberico, E.; Baumann, W.; Drexler, H.-J.; Junge, H.; Gladiali, S.; Beller, M. Nature 2013, 495, 85 .

[42] Li, H.; Hall, M. B. J. Am. Chem. Soc. 2015, 137, 12330.

[43] Jing, Y.; Chen, X.; Yang, X. J. Organomet. Chem. 2016, 820, 55.

[44] Sinha, V.; Trincado, M.; Grützmacher, H.; de Bruin, B., J. Am. Chem. Soc. 2018, 140, 13103.

[45] Kuriyama, W.; Matsumoto, T.; Ogata, O.; Ino, Y.; Aoki, K.; Tanaka, S.; Ishida, K.; Kobayashi, T.; Sayo, N.; Saito, T. Org. Process. Res. Dev. 2012, 16, 166.

[46] (a) Bertoli, M.; Choualeb, A.; Lough, A. J.; Moore, B.; Spasyuk, D.; Gusev, D. G. Organometallics 2011, 30, 3479.

(b) Nielsen, M.; Kammer, A.; Cozzula, D.; Junge, H.; Gladiali, S.; Beller, M. Angew. Chem., Int. Ed. Engl. 2011, 50, 9593.

[47] Lei, M.; Pan, Y.; Ma, X. Eur. J. Inorg. Chem. 2015, 2015, 794.

[48] Alberico, E.; Lennox, A. J.; Vogt, L. K.; Jiao, H.; Baumann, W.; 
Drexler, H. J.; Nielsen, M.; Spannenberg, A.; Checinski, M. P.; Junge, H.; Beller, M. J. Am. Chem. Soc. 2016, 138, 14890.

[49] Yang, X. ACS Catal. 2014, 4, 1129.

[50] Monney, A.; Barsch, E.; Sponholz, P.; Junge, H.; Ludwig, R.; Beller, M. Chem. Commun. 2014, 50, 707.

[51] Hu, P.; Diskin-Posner, Y.; Ben-David, Y.; Milstein, D. ACS Catal. 2014, 4, 2649

[52] Heim, L. E.; Schlörer, N. E.; Choi, J. H.; Prechtl, M. H. Nat. Commun. 2014, 5, 3621.

[53] Crabtree, R. H. New J. Chem. 2011, 35, 18.

[54] (a) Crabtree, R. H. Chem. Rev. 2017, 117, 9228. (b) Grützmacher, H., Angew. Chem., Int. Ed. 2008, 47, 1814.

[55] Kothandaraman, J.; Kar, S.; Goeppert, A.; Sen, R.; Prakash, G. K. S. Top. Catal. 2018, 61, 542.

[56] Heim, L. E.; Thiel, D.; Gedig, C.; Deska, J.; Prechtl, M. H. G. Angew. Chem., Int. Ed. 2015, 54, 10308.

[57] Kothandaraman, J.; Goeppert, A.; Czaun, M.; Olah, G. A.; Prakash, G. K. S. J. Am. Chem. Soc. 2016, 138, 778.

[58] Van de Watering, F. F.; Lutz, M.; Dzik, W. I.; de Bruin, B.; Reek, J. N. H. Chem CatChem 2016, 8, 2752.

[59] Shinoda, S.; Yamakawa, T. J. Chem. Soc., Chem. Commun. 1990, 1511.

[60] Shinoda, S.; Ohnishi, T.; Yamakawa, T. Catal. Sur. Asia 1997, 1, 25.

[61] Robles-Dutenhefnera, P. A.; Mourab, E. M.; Gamac, G. J. J. Mol. Catal. A Chem. 2000, 164, 39.

[62] Campos, J.; Sharninghausen, L. S.; Manas, M. G.; Crabtree, R. H. Inorg. Chem. 2015, 54, 5079.

[63] Manas, M. G.; Campos, J.; Sharninghausen, L. S.; Lin, E.; Crabtree,
R. H. Green Chemistry 2015, 17, 594.

[64] Fujita, K.; Kawahara, R.; Aikawa, T.; Yamaguchi, R. Angew. Chem. 2015, 127, 9185.

[65] Prichatz, C.; Alberico, E.; Baumann, W.; Junge, H.; Beller, M. ChemCatChem 2017, 9, 1891.

[66] Shen, Y.; Zhan, Y.; Li, S.; Ning, F.; Du, Y.; Huang, Y.; He, T.; Zhou, X. Chem. Sci. 2017, 8, 7498 .

[67] Morton, D.; Cole-Hamilton, D. J. J. Chem. Soc. Chem. Commun. 1987, 0,248

[68] Zhan, Y. L.; Shen, Y. B.; Li, S. P.; Yue, B. H.; Zhou, X. C. Chin. Chem. Lett. 2017, 28, 1353.

[69] Chai, H.-N.; Liu,B.; Liu, A.-Q.; Yu, K. J. Mol. Catal 2018, 32, 481 (in Chinese) (柴会宁，刘波，刘爱芹，喻琨，分子催化， 2018, 32,481.)

[70] Alberico, E.; Sponholz, P.; Cordes, C.; Nielsen, M.; Drexler, H. J.; Baumann, W.; Junge, H.; Beller, M. Angew. Chem., Int. Ed. Engl. 2013, 52, 14162

[71] Bielinski, E. A.; Förster, M.; Zhang, Y.; Bernskoetter, W. H.; Hazari, N.; Holthausen, M. C. ACS Catal. 2015, 5, 2404.

[72] Wakizaka, M.; Matsumoto, T.; Tanaka, R.; Chang, H.-C. Nat. Commun. 2016, 7, 12333.

[73] Eisenstein, O.; Crabtree, R. H. New J. Chem. 2013, 37, 21.

[74] Andérez-Fernández.M; Vogt, L. K.; Fischer, S.; Zhou, W.; Jiao, H.; Garbe, M.; Elangovan, S.; Junge, K.; Junge, H.; Ludwig, R.; Beller, M. Angew. Chem., Int. Ed. Engl. 2017, 56, 559.

[75] Wei, Z.; de Aguirre, A.; Junge, K.; Beller, M.; Jiao, H. J. Catal. Sci. Technol. 2018, 8, 3649 . 